\title{
Bioequivalence Study of Hydrocortisone Tablets while Secretion of Endogenous Cortisol Suppressed
}

\author{
Tae-Suk OK ${ }^{1}$, Kyoung-Jin LeE ${ }^{2}$, and Young-Hee SHIN ${ }^{1 *}$ \\ ${ }^{1}$ College of Pharmacy, Kyungsung University, Busan 608-736, Korea \\ ${ }^{2}$ Ligand Pharmaceuticals, San Diego, CA 92121, USA
}

(Received July 9, 2008; Revised August 4, 2008; Accepted August 12, 2008)

\begin{abstract}
The purpose of this study was to evaluate the bioequivalence of the test (Daewoo Hydrocortisone 10 mg, Daewoo Pharm. Co., Busan, Korea) and reference (Jenapharm Hydrocortisone 10 mg, JayTech Biogen, Seoul, Korea) hydrocortisone tablets. Twenty-four healthy male Korean volunteers were divided into two groups with a randomized $2 \times 2$ cross-over design. In order to suppress the endogenous cortisol secretion, a single oral dose of Dexamethasone $(4 \mathrm{mg})$ was administered $10 \mathrm{hr}$ prior to hydrocortisone administration. Blood samples were withdrawn for $10 \mathrm{hr}$ at the predetermined intervals after a single oral dose of hydrocortisone (20 $\mathrm{mg}$ ). The serum concentration of hydrocortisone was analyzed by HPLC/UV using a column switching method after liquid-liquid extraction process. The pharmacokinetic parameters $\left(\mathrm{AUC}_{0 \sim 10 \mathrm{hr}}, \mathrm{C}_{\max }\right.$, and $\left.\mathrm{T}_{\max }\right)$ of the test and reference hydrocortisone tablets were determined while the secretion of endogenous cortisol was being suppressed. The pharmacokinetic parameters of the test tablet were not statistically different from those of the reference tablet at $\alpha$ value was 0.05 . The $90 \%$ confidence intervals for the average ratio (test/reference) of $\mathrm{AUC}_{0 \sim 10 \mathrm{hr}}$ and $\mathrm{C}_{\max }$ were within the Korea Food and Drug Administration acceptance range of 0.80-1.25 (0.89 0.99 and $0.86 \sim 0.99$ for $\mathrm{AUC}_{0 \sim 10 \mathrm{hr}}$ and $\mathrm{C}_{\max }$, respectively). Therefore it was concluded that the test tablet, Daewoo Hydrocortisone tablet was bioequivalent to the reference tablet, Jenapharm Hydrocortisone tablet.
\end{abstract}

Keywords: Bioequivalence, Hydrocortisone, Suppression of endogenous cortisol, Pharmacokinetics

\section{INTRODUCTION}

Cortisol is one of steroid hormones produced by the adrenal cortex, and is called glucocorticoid because it shows an important effect on the metabolism of glucose and other organic nutrients (Vander et al., 1990a). The secretion of cortisol is increased in the response to stimuli such as physical trauma, prolonged exposure to cold, heavy exercise, shock, infection, pain, and emotional stresses. The elevated cortisol plasma concentration increases the organic metabolism, enhances the vascular activity, and inhibits the inflammation and immune response, thus it has been used as a tool in the treatment of allergy, arthritis, and other inflammatory diseases. The cortisol plasma concentration profile follows diurnal variation, the highest plasma level is found in the early morning, and the lowest plasma level, in the middle

\section{${ }^{*}$ Corresponding author}

Tel: 82-51-620-4886,

E-mail: yhshin@ks.ac.kr of night (Vander et al., 1990b; Vander et al., 1990c).

Hydrocortisone is the synthetic cortisol, and is frequently prescribed for allergies and inflammation. The purpose of this study was to evaluate the bioequivalence of the test (Daewoo Hydrocortisone $10 \mathrm{mg}$, Daewoo Pharmaceuticals, Busan, Korea) and reference (Jenapharm Hydrocortisone $10 \mathrm{mg}$, JayTech Biogen, Seoul, Korea) hydrocortisone tablets, according to the guidelines of Korea Food and Drug Administration (Korea Food and Drug Administration, 2005). Twenty-four healthy male Korean volunteers were divided into two groups with a randomized $2 \times 2$ cross-over design. The pharmacokinetic parameters $\left(\mathrm{AUC}_{0 \sim 10 \mathrm{hr}}, \mathrm{C}_{\max }\right.$, and $\left.\mathrm{T}_{\max }\right)$ of the test and reference hydrocortisone tablets were determined while the secretion of endogenous cortisol was suppressed with a single oral dose of dexamethasone $(4 \mathrm{mg})$ (Goehl et al., 1980; Toothaker and Welling, 1982). The statistical analysis of the pharmacokinetic parameters was performed to determine whether the test and reference tablets were comparable. 


\section{MATERIALS AND METHODS}

\section{Materials}

Hydrocortisone, prednisolone, sodium dihydrogen phosphate, sodium hydrogen phosphate were purchased from Sigma Aldrich (St. Louis, MO, USA). HPLC grade acetonitrile, methanol, and water were obtained from Merck Co. (Darmstadt, Germany). The test (Lot No. 501) and reference (Lot No. 42702) hydrocortisone $10 \mathrm{mg}$ tablets were supplied from Daewoo Pharmaceuticals, Busan, Korea, and JayTech Biogen, Seoul, Korea, respectively. Dexamethasone $(0.5 \mathrm{mg})$ tablets were obtained from Yuhan Medica (Kunpo, Korea).

\section{Sample analysis}

\section{Preparation of calibration standards}

A stock solution of hydrocortisone was prepared in methanol at $1 \mathrm{mg} / \mathrm{mL}$, and used for preparation of calibration standards. "Cortisol-free" human serum was obtained after a single oral dose of dexamethasone (4 $\mathrm{mg}$ ) in healthy Korean subjects. The collected serum with dexamethasone administration was used as blank serum. The blank serum was spiked with the diluted hydrocortisone methanol stock solution, and the calibration standards were prepared in the concentration ranges of 10 to $1000 \mathrm{ng} / \mathrm{mL}$.

\section{Sample extraction}

Two hundred micro liters of experimental serum and calibration standard samples were combined with $400 \mu \mathrm{L}$ of acetonitrile containing prednisolone (internal standard, $200 \mathrm{ng} / \mathrm{mL}$ ). The mixture was vortexed for $1 \mathrm{~min}$, followed by centrifugation at $10,000 \mathrm{rpm}$ for $5 \mathrm{~min}$. The organic phase $(400 \mu \mathrm{L})$ was transferred and evaporated in a speed-vac, and the residue was reconstituted with 100 $\mu \mathrm{L}$ of the mobile phase 1 .

\section{Chromatographic condition}

Hydrocortisone and prednisolone were analyzed by HPLC/UV using a switching column method, following the liquid-liquid extraction (Okumura et al., 1995; van der Hoeven et al., 1997; Kim et al., 2005). A Capcell pak MF $\mathrm{C}_{8}(4.6 \times 50 \mathrm{~mm})$ and a Capcell Pak MF $\mathrm{C}_{18}(2.0 \times 35 \mathrm{~mm})$ were used as a pre-column and a trap-column (Shiseido, Tokyo, Japan), respectively. A Synergi $4 \mu$-Fusion RP column $(4.6 \times 250 \mathrm{~mm})$ was used as an analytical column (Phenomenex, Torrance, CA, USA). Hydrocortisone and prednisolone were retained in pre-column and trap-column with the mobile phase 1 ( $2 \mathrm{mM}$ citrate buffer $(\mathrm{pH}$ 6.5): acetonitrile $(85: 15, \mathrm{v} / \mathrm{v} \%)$ ) at a flow rate of $0.5 \mathrm{~mL} /$ min, and eluted through the analytical column with the mobile phase 2 (water: acetonitrile $(57: 43, \mathrm{v} / \mathrm{v} \%)$ ) at a flow rate of $0.7 \mathrm{~mL} / \mathrm{min}$. The column temperature was maintained at $40^{\circ} \mathrm{C}$, and both hydrocortisone and prednisolone were detected at $254 \mathrm{~nm}$.

\section{Analytical method validation}

The linearity of the analytical method was examined over the standard concentration ranges (10 1000 $\mathrm{ng} / \mathrm{mL})$. The calibration equation was constructed by fitting the normalized peak area (ratio of hydrocortisone peak area to internal standard peak area) as a function of hydrocortisone concentration in each calibration standard, and was obtained from least-square linear regression.

Intra-day and inter-day precision and accuracy were calculated at all the calibration standard concentrations. Intra-day precision was assessed from 5 consecutive determinations within a single day run, and inter-day precision was determined from 5 consecutive day determinations, and the precision results were presented as the coefficient of variation (CV \%). Intra-day and inter-day accuracy was examined by calculating the agreement between the measured concentrations and the nominal concentrations, and the accuracy results was presented in average \pm standard deviation (S.D.).

\section{Pharmacokinetics of hydrocortisone in Korean \\ Measurement of endogenous cortisol concentrations}

Healthy male Korean volunteers were enrolled for measurement of the endogenous cortisol concentration. Physical and biological examinations were performed for the enrolled volunteers at Wallace Memorial Baptist Hospital (Busan, Korea), and the volunteers who met the criteria of subject selection were selected for the study. The selected subjects $(n=8)$ were informed with a written document detailing the purpose, procedure, and risks of the study, and signed the consent form prior to the study initiation. The average age of the subjects was 24.5 $( \pm 3.8)$ years old, and the average weight was 63.8 $( \pm 5.7) \mathrm{kg}$.

All the subjects were refrained from taking all medications including antibiotics and analgesics, smoking and beverages with xanthines for 10 days before the study. On Day 1 of the study, the blood samples $(5 \mathrm{~mL})$ were withdrawn from the upper arm vein of the subjects at 8:00 am, and thereafter every two hours till 10:00 pm. During the day, all the subjects were provided with a standard meal for lunch and dinner. The withdrawn blood samples were centrifuged at $3,000 \mathrm{rpm}$ for $10 \mathrm{~min}$, and serum was separated and stored at $-70^{\circ} \mathrm{C}$ until analysis. 


\section{Suppression of endogenous cortisol secretion}

Following the last blood withdrawal at 10:00 pm of Day 1 , all the subjects received a single oral dose of dexamethasone (4 mg) (Derendorf et al., 1991; Mollmann et al., 1991). The next day morning the blood samples $(5 \mathrm{~mL})$ were withdrawn from the upper arm vein at 8:00 am and thereafter every two hours till 8:00 pm. During the study all the subjects were hospitalized in order to limit and monitor their physical activity, meals, smoking, and beverages with xanthines. The blood samples were centrifuged at 3,000 rpm for $10 \mathrm{~min}$ and serum was separated and stored at $-70^{\circ} \mathrm{C}$ until analysis.

\section{Bioequivalence study of hydrocortisone in Korean}

The pharmacokinetic parameters of the test and reference hydrocortisone tablets were determined in 24 healthy Korean subjects. Physical and biological examinations were performed for the enrolled volunteers at Wallace Memorial Baptist Hospital (Busan, Korea), and the volunteers who met the criteria of subject selection were selected for the study. The selected subjects were informed with a written document detailing the purpose, procedure, and risks of the study, and signed the consent form prior to the study initiation. Twenty-four healthy male Korean volunteers were divided into two groups with a randomized $2 \times 2$ cross-over design. The night before the study, all the subjects were hospitalized at 7:00 pm and received a single oral dose of dexamethasone (4 mg) at 10:00 pm and were fasted till the next day noon. During the study the subjects were refrained from physical activity, smoking, and beverages containing xanthines. The blank blood samples were withdrawn at 7:00 am the next day morning ( $0 \mathrm{hr})$, and a single oral dose of hydrocortisone $(20 \mathrm{mg})$ was administered to the first subject at 8:00 am with a two-minute interval between subjects. Blood samples $(5 \mathrm{~mL})$ were withdrawn $0.33,0.66$, $1,1.5,2,2.5,3,4,6,8$, and $10 \mathrm{hr}$ post drug administration. The blood samples were centrifuged at $3,000 \mathrm{rpm}$ for $10 \mathrm{~min}$ and serum was separated and stored at $-70^{\circ} \mathrm{C}$ until analysis.

The pharmacokinetic parameters of the test and reference tablets such as maximum serum concentration $\left(C_{\max }\right)$, time to reach the $\mathrm{C}_{\max }\left(\mathrm{T}_{\max }\right)$, and area under the serum concentration-time curve $\left(\mathrm{AUC}_{0 \sim 10 \mathrm{hr}}\right)$ were determined using K-Be Test program (Korea Food Drug Administration) and Equiv Test version 1.0 (Statistical Solution Ltd., 1998). $A \cup \mathrm{C}_{0 \sim 10 \mathrm{hr}}$ and $\mathrm{C}_{\max }$ were logarithmically transformed to stabilize the variance and to obtain the symmetric data distribution. Bioequivalence between the test and reference tablets were assessed based on the statistical analysis (ANOVA) of In $\left(\mathrm{AUC}_{0 \sim 10 \mathrm{hr}}\right)$, In $\left(\mathrm{C}_{\max }\right)$ and $T_{\max }$.

\section{RESULTS AND DISCUSSION}

\section{Chromatography of hydrocortisone}

The blank serum was obtained after administration of dexamethasone and its chromatogram was shown in Fig. 1(A). Cortisol was found in the blank serum, and the peak area of the baseline cortisol was subtracted from the

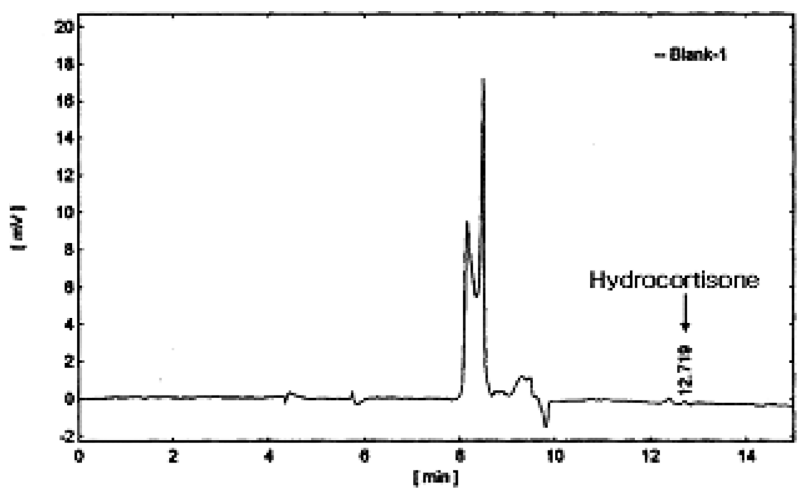

(A)

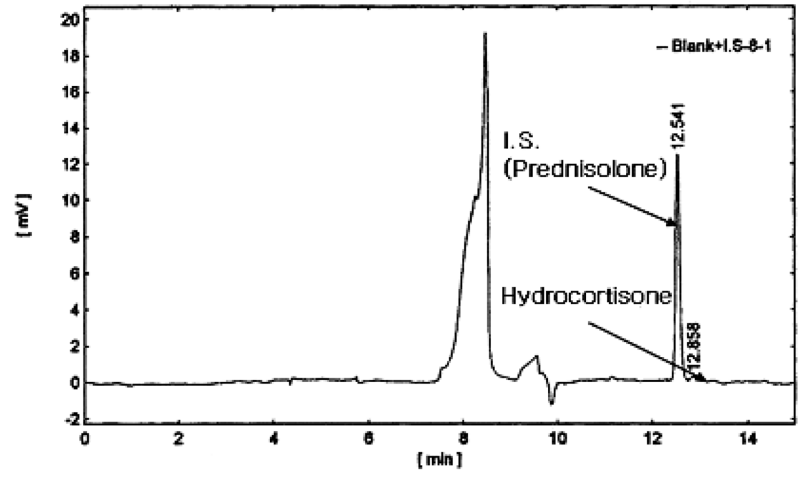

(B)

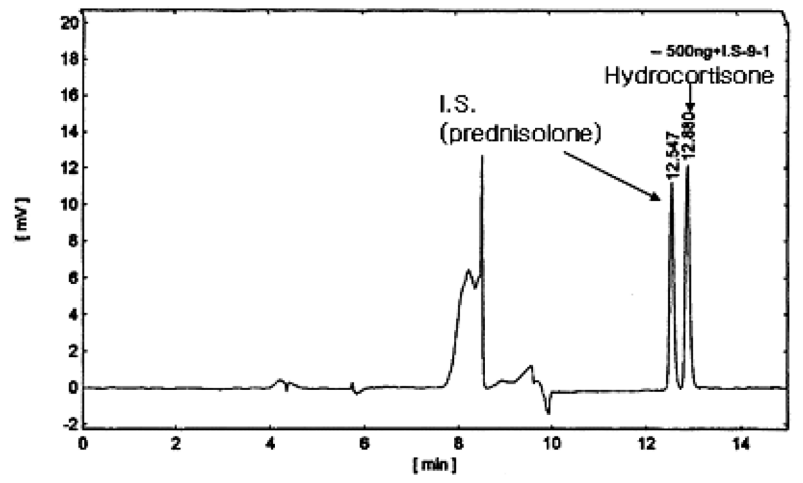

(C)

Fig. 1. Exemplary chromatograms of "cortisol-free" blank serum (A), a serum spiked with prednisolone (I.S.) (B), and a serum spiked with prednisolone (I.S.) and hydrocortisone (C). 
peak area of standards and pharmacokinetic samples for quantification. Chromatograms of serum spiked with hydrocortisone and prednisolone were shown in Fig. 1(B) and $(\mathrm{C})$. Hydrocortisone and prednisolone were eluted at 12.8 and $12.5 \mathrm{~min}$, respectively.

\section{Sensitivity and linearity}

The peak areas of hydrocortisone and internal standard in calibration standards were determined by automated integration. The lower limit of quantification (LOQ) was $10 \mathrm{ng} / \mathrm{mL}$ with a signal-to-noise ratio of 10 . The calibration equation of this method was $y=0.23 x-0.30\left(R^{2}=\right.$ 0.999 ), where $y$ is the normalized peak area of hydrocortisone and $\mathrm{x}$, the standard concentration.

\section{Accuracy and Precision}

The results of intra-day and inter-day precision and accuracy were presented in Table I. The coefficient of variations of intra-day and inter-day precision were less than $15 \%$ except for the lower limit of quantification, of which were 19.02 and $16.23 \%$ respectively. Intra-day and inter-day accuracy ranged from $96.6 \%$ to $115.1 \%$.

\section{Measurement of endogenous cortisol concentra- tions}

The endogenous cortisol concentrations were monitored over the $14 \mathrm{hr}$ period in 8 healthy Korean subjects for the first time. As shown in Fig. 2, the endogenous cortisol concentration followed a diurnal pattern with a peak at 8:00 am (155.8 \pm 44.6$) \mathrm{ng} / \mathrm{mL}$ and a nadir at 10:00 pm $(28.5 \pm 7.0 \mathrm{ng} / \mathrm{mL})$, which were similar to the reported values of other ethnic groups (Hardman et al., 2001). A single oral dose of dexamethasone $(4 \mathrm{mg})$ suppressed the secretion of endogenous cortisol to $11.0 \pm 0.6 \mathrm{ng} / \mathrm{mL}$, which was lower than the nadir of cortisol diurnal pattern (Fig. 2). The suppression effect of dexamethasone on the endogenous cortisol secretion lasted during the next day from 8:00 am till 8:00 pm, and this finding was referred to

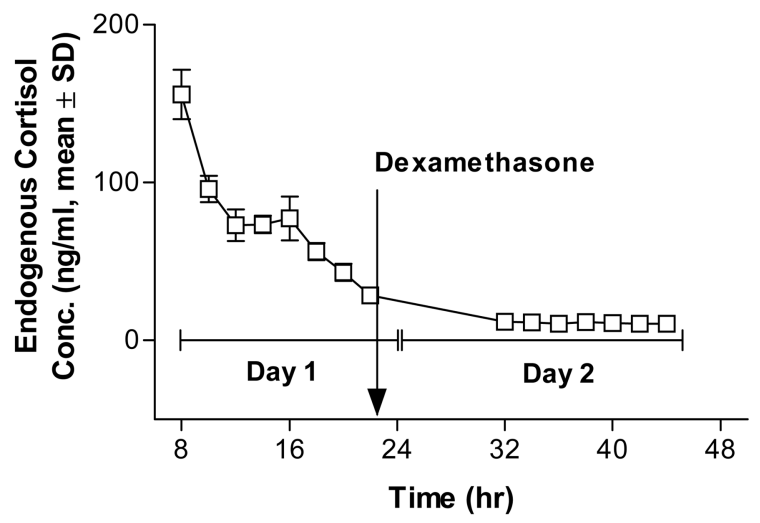

Fig. 2. Endogenous serum cortisol concentration changes before and after administration of a single oral dose of dexamethasone $(4 \mathrm{mg})$ in healthy Korean subjects $(n=8)$.

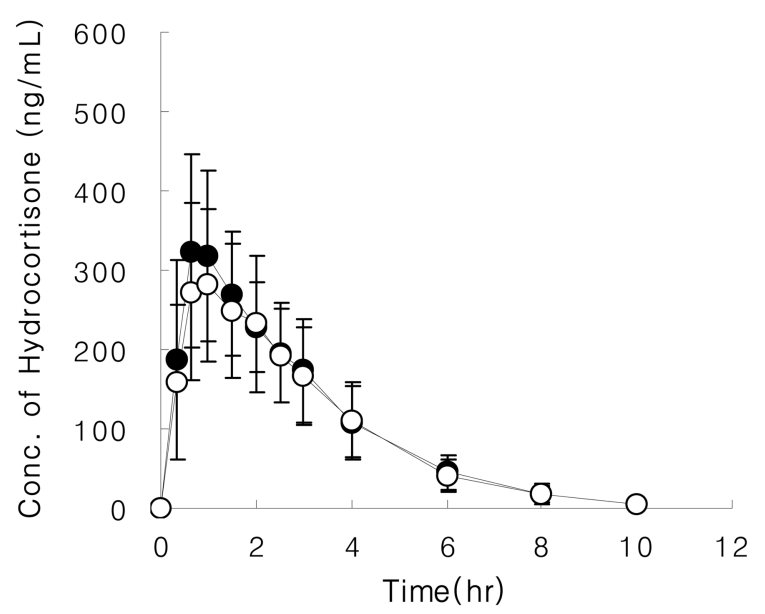

Fig. 3. Serum concentration-time profiles of hydrocortisone following oral administration of Daewoo Hydrocortisone (test, open circle) and Jenapharm Hydrocortisone (reference, closed circle) at a dose of $20 \mathrm{mg}$ in healthy Korean subjects while suppressing the endogenous cortisol with a single oral dose of dexamethasone (4 mg). Data was represented as Average \pm S.D. $(n=24)$.

the pharmacokinetic study of the test and reference hydrocortisone tablets.

Table I. Intra-day and inter-day precision and accuracy for hydrocortisone in human serum $(\mathrm{n}=5)$.

\begin{tabular}{|c|c|c|c|c|}
\hline \multirow{2}{*}{$\begin{array}{l}\text { Conc. } \\
\text { (ng/mL) }\end{array}$} & \multicolumn{2}{|c|}{ Precision (CV \%) } & \multicolumn{2}{|c|}{ Accuracy (\%, Average \pm S.D.) } \\
\hline & Intra-Day & Inter-Day & Intra-Day & Inter-Day \\
\hline 10 & 19.02 & 16.23 & $97.58 \pm 16.04$ & $115.07 \pm 16.50$ \\
\hline 20 & 12.89 & 13.78 & $101.05 \pm 12.17$ & $110.82 \pm 14.37$ \\
\hline 40 & 7.96 & 3.40 & $105.02 \pm 8.10$ & $102.04 \pm 3.35$ \\
\hline 100 & 3.17 & 4.45 & $104.93 \pm 3.29$ & $100.87 \pm 4.43$ \\
\hline 200 & 1.79 & 4.29 & $99.09 \pm 1.77$ & $97.16 \pm 4.13$ \\
\hline 500 & 3.97 & 6.19 & $101.89 \pm 4.03$ & $96.61 \pm 5.96$ \\
\hline 1000 & 2.54 & 7.58 & $103.95 \pm 2.63$ & $97.35 \pm 7.37$ \\
\hline
\end{tabular}




\section{Pharmacokinetics of hydrocortisone after oral administration}

In order to suppress the secretion of endogenous cortisol a single oral dose of dexamethasone $(4 \mathrm{mg}$ ) was administered $10 \mathrm{hr}$ prior to hydrocortisone administration. The average $( \pm$ S.D.) serum concentration-time profiles of the test and reference hydrocortisone tablets in 24 healthy Korean subjects were shown in Fig. 3 while the secretion of endogenous cortisol was suppressed.

The bioequivalence between test and reference tablets was examined according to the Bioequivalence criteria of Korea Food and Drug Administration (KFDA). The actual and logarithmically transformed pharmacokinetic parameters were presented in Table II. No significant carry-over effect was observed between the periods in these parameters (statistical analysis results not shown). The statistical results of bioequivalence test between the test and reference tablets were presented in Table III. The percent differences between test and reference tablets in averages of $\mathrm{AUC}_{0 \sim 10 \mathrm{hr}}$ and $\mathrm{C}_{\max }$ were $-6.44 \%$ and $-7.77 \%$, respectively, and the differences were within the acceptance criteria $( \pm 20 \%)$. Pharmacokinetic parameters of the test tablet were not statistically different from those of the reference tablet at $\alpha=0.05$ and $\beta=0.2$. The $90 \%$ confidence intervals for the average ratio (test/reference) of $A U C_{0 \sim 10 h r}$ and $C_{\max }$ were within the KFDA acceptance range of $0.80-1.25(0.89 \sim 0.99$ and $0.86 \sim 0.99$ for $\mathrm{AUC}_{0 \sim 10 \mathrm{hr}}$ and $\mathrm{C}_{\max }$, respectively). The percent difference of $T_{\max }$ was within the acceptance criteria, however the $90 \%$ confidence interval for the average ratio of $T_{\max }$ fell

Table II. Phamacokinetic parameters in normal and logarithmic scales for each volunteer after oral administration of Jenapharm Hydrocortisone (reference) and Daewoo Hydrocortisone (test) tablets.

\begin{tabular}{|c|c|c|c|c|c|c|c|c|c|c|}
\hline \multirow{4}{*}{ Subject_\# } & \multicolumn{10}{|c|}{ Parameters } \\
\hline & \multicolumn{4}{|c|}{$\mathrm{AUC}_{0 \sim 10 \mathrm{hr}}\left(\mathrm{ng}^{*} \mathrm{hr} / \mathrm{mL}\right)$} & \multicolumn{4}{|c|}{$\mathrm{C}_{\max }(\mathrm{ng} / \mathrm{mL})$} & \multicolumn{2}{|c|}{$\mathrm{T}_{\max }(\mathrm{hr})$} \\
\hline & \multicolumn{2}{|c|}{ Reference } & \multicolumn{2}{|c|}{ Test } & \multicolumn{2}{|c|}{ Reference } & \multicolumn{2}{|c|}{ Test } & \multirow{2}{*}{$\begin{array}{c}\text { Reference } \\
\text { Actual }\end{array}$} & \multirow{2}{*}{$\begin{array}{c}\text { Test } \\
\text { Ln (actual) }\end{array}$} \\
\hline & Actual & Ln (actual) & Actual & Ln (actual) & Actual & Ln (actual) & Actual & Ln (actual) & & \\
\hline 1 & 680.0 & 6.5 & 579.3 & 6.4 & 401.0 & 6.0 & 327.4 & 5.8 & 0.7 & 0.7 \\
\hline 2 & 1182.1 & 7.1 & 1196.1 & 7.1 & 467.1 & 6.2 & 389.1 & 6.0 & 0.7 & 0.7 \\
\hline 3 & 1287.6 & 7.2 & 1296.2 & 7.2 & 303.9 & 5.7 & 494.8 & 6.2 & 2.5 & 2.0 \\
\hline 4 & 1108.3 & 7.0 & 1079.4 & 7.0 & 388.0 & 6.0 & 357.5 & 5.9 & 1.5 & 1.0 \\
\hline 5 & 1302.1 & 7.2 & 944.1 & 6.9 & 429.4 & 6.1 & 318.9 & 5.8 & 0.7 & 0.7 \\
\hline 6 & 881.2 & 6.8 & 1021.8 & 6.9 & 395.0 & 6.0 & 474.9 & 6.2 & 1.0 & 0.7 \\
\hline 7 & 1248.8 & 7.1 & 1090.3 & 7.0 & 362.0 & 5.9 & 404.5 & 6.0 & 0.7 & 1.5 \\
\hline 8 & 1453.7 & 7.3 & 1315.3 & 7.2 & 542.8 & 6.3 & 488.2 & 6.2 & 1.0 & 1.0 \\
\hline 9 & 1161.7 & 7.1 & 990.8 & 6.9 & 375.9 & 5.9 & 313.3 & 5.8 & 0.7 & 0.7 \\
\hline 10 & 1058.1 & 7.0 & 951.7 & 6.9 & 313.9 & 5.8 & 320.2 & 5.8 & 2.5 & 2.0 \\
\hline 11 & 637.0 & 6.5 & 560.6 & 6.3 & 286.4 & 5.7 & 292.0 & 5.7 & 0.3 & 0.3 \\
\hline 12 & 1342.4 & 7.2 & 1237.8 & 7.1 & 368.7 & 5.9 & 371.5 & 5.9 & 0.7 & 1.5 \\
\hline 13 & 892.3 & 6.8 & 1079.8 & 7.0 & 409.5 & 6.0 & 309.1 & 5.7 & 1.0 & 3.0 \\
\hline 14 & 1105.8 & 7.0 & 1106.3 & 7.0 & 470.7 & 6.2 & 384.6 & 6.0 & 0.7 & 0.7 \\
\hline 15 & 940.9 & 6.9 & 892.8 & 6.8 & 243.3 & 5.5 & 273.6 & 5.6 & 1.0 & 1.0 \\
\hline 16 & 1005.7 & 6.9 & 1058.4 & 7.0 & 558.8 & 6.3 & 305.0 & 5.7 & 0.7 & 2.0 \\
\hline 17 & 1176.5 & 7.1 & 693.6 & 6.5 & 454.5 & 6.1 & 339.4 & 5.8 & 1.0 & 0.7 \\
\hline 18 & 897.6 & 6.8 & 911.6 & 6.8 & 393.4 & 6.0 & 389.9 & 6.0 & 0.7 & 0.7 \\
\hline 19 & 403.4 & 6.0 & 444.0 & 6.1 & 353.0 & 5.9 & 313.5 & 5.8 & 0.3 & 0.7 \\
\hline 20 & 875.7 & 6.8 & 782.6 & 6.7 & 329.2 & 5.8 & 252.6 & 5.5 & 1.0 & 2.5 \\
\hline 21 & 1249.7 & 7.1 & 1142.9 & 7.0 & 415.5 & 6.0 & 451.8 & 6.1 & 0.7 & 1.0 \\
\hline 22 & 810.4 & 6.7 & 893.3 & 6.8 & 299.5 & 5.7 & 258.1 & 5.6 & 3.0 & 3.0 \\
\hline 23 & 762.0 & 6.6 & 758.2 & 6.6 & 322.7 & 5.8 & 358.1 & 5.9 & 1.0 & 0.7 \\
\hline 24 & 1359.5 & 7.2 & 1197.6 & 7.1 & 425.3 & 6.1 & 398.7 & 6.0 & 1.0 & 1.0 \\
\hline 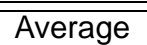 & 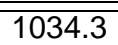 & 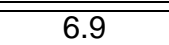 & $\begin{array}{l}967.7 \\
\end{array}$ & 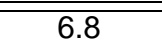 & $\overline{3887.9}$ & $\overline{5.9}$ & $\begin{array}{l}357.8 \\
\end{array}$ & $\overline{\overline{5.9}}$ & $\overline{c 1.0}$ & 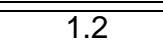 \\
\hline SD & 261.9 & 0.3 & 234.8 & 0.3 & 77.2 & 0.2 & 69.4 & 0.2 & 0.7 & 0.8 \\
\hline
\end{tabular}


Table III. Statistical results of bioequivalence test between the test and reference hydrocortisone tablets.

\begin{tabular}{lcccc}
\hline \multicolumn{1}{c}{ Parameters } & Criteria for bioequivalence & $\mathrm{AUC}_{0 \sim 10 \mathrm{hr}}$ & $\mathrm{C}_{\max }$ & $\mathrm{T}_{\max }$ \\
\hline Percent difference of averages & $\pm 20 \%$ & -6.44 & -7.77 & 18.86 \\
$\mathrm{~F}(1,22, \alpha=0.05)$ & $<4.30$ & 1.47 & 0.79 & 0.22 \\
$1-\beta$ & $>0.8$ & $>0.9$ & $>0.9$ & $<0.6$ \\
Point estimate of the average ratio $(\delta)$ & $\mathrm{NA}$ & 0.94 & 0.92 & 1.17 \\
Confidence interval at $\alpha=0.05$ & $0.8 \leq \delta \leq 1.25$ & $0.89 \leq \delta \leq 0.99$ & $0.86 \leq \delta \leq 0.99$ & $0.99 \leq \delta \leq 1.39$ \\
\hline
\end{tabular}

outside of the acceptance range and the power was less than $60 \%$. The statistical analysis result of $\mathrm{T}_{\max }$ was not considered to be critical for a bioequivalence study, therefore it was concluded that the test tablet, Daewoo Hydrocortisone tablet was bioequivalent to the reference tablet, Jenapharm Hydrocortisone.

\section{CONCLUSION}

An HPLC/UV analytical method of hydrocortisone was developed using a column switching method, and was validated in terms of linearity, accuracy, and precision. The diurnal pattern of the endogenous cortisol in serum was monitored and reported in Koreans for the first time. The pharmacokinetic parameters of the test tablet were not statistically different from those of the reference tablet at $\alpha=0.05$. The $90 \%$ confidence intervals for the average ratio (test/reference) of $\mathrm{AUC}_{0 \sim 10 \mathrm{hr}}$ and $\mathrm{C}_{\max }$ were within the KFDA acceptance range, 0.80-1.25 (0.89 0.99 and $0.86 \sim 0.99$ for $\mathrm{AUC}_{0 \sim 10 \mathrm{hr}}$ and $\mathrm{C}_{\max }$, respectively). Therefore it was concluded that the test tablet, Daewoo Hydrocortisone tablet was bioequivalent to the reference tablet, Jenapharm Hydrocortisone tablet.

\section{ACKNOWLEDGEMENTS}

This research was supported by Daewoo Pharm. Co. (Busan, Korea).

\section{REFERENCES}

Derendorf, H., Mollmann, H., Barth, J., Mollmann, C., Tunn, S. and Krieg, M. (1991). Pharmacokinetics and oral bioavailability of hydrocortisone. J. Clin. Pharmacol., 31, 473-476.

Goehl, T.J., Sundaresan, G.M., Hunt, J.P., Prasad, V.K., Toothaker, R.D. and Welling, P.G. (1980). Suppression of endogenous hydrocortisone with dexamethasone. J. Pharm. Sci. 69, 1409-1410.
Hardman, J.G., Limbird, L.E. and Gilman, A.G. (2001). Goodman \& Gilman's The Pharmacological basis of Therapeutics, $10^{\text {th }}$ edition, McGraw-Hill Professional, pp 1655.

Kim, C.-K., Yeon, K. J., Ban, E., Hyun, M.-J., Kim, J.-K., Kim, M.-K., Jin, S.-E. and Park, J.-S. (2005). Narrow-bore high performance liquid chromatographic method for the determination of cetirizine in human plasma using column switching. J. Pharm. Biomed. Anal., 37, 603-609.

Korea Food and Drug Administration. (2005). Korea guidelines for bioequivalence test 2005-31. Seoul, Korea.

Mollmann, H., Barth, J., Mollmann, C., Tunn, S., Krieg, M. and Derendorf, H. (1991). Pharmacokinetics and rectal bioavailability of hydrocortisone acetate. J. Pharm. Sci., 80, 835-836.

Okumura, T., Nakajima, Y., Takamastu, T. and Matsuoka, M. J. (1995). Column-switching high-performance liquid chromatographic system with a laser-induced fluorimetric detector for direct, automated assay of salivary cortisol. Chromatogr. B: Biomed. Sci. Appl., 670, 11-20.

Toothaker, R. D. and Welling, P.G. (1982). Effect of dose size on the pharmacokinetics of intravenous hydrocortisone during endogenous hydrocortisone suppression. J. Pharmacokinet. Biopharm., 10, 147-156.

Vander Hoeven, R. A., Hofte, A. J., Frenay, M., Irth, H., Tjaden, U. R., vander Greef, J., Rudolphi, A., Boos, K.S., Varga, G. M. and Edholm, L. E. (1997). Liquid chromatography-mass spectrometry with on-line solid-phase extraction by a restricted-access $\mathrm{C} 18$ precolumn for direct plasma and urine injection. J. Chromatogr. A., 762, 193-200.

Vander, A. J., Sherman, J. H. and Luciano, D. S. (1990a). The mechanisms of body function. In Human Physiology (Nunes, I., Schanck, D.T. and Bradley, J.W. Ed.), pp. 255-282. International edition, McGraw-Hill Publishing Company, Columbus, $\mathrm{OH}$.

Vander, A. J., Sherman, J. H. and Luciano, D. S. (1990b). The mechanisms of body function. In Human Physiology (Nunes, I., Schanck, D.T. and Bradley, J.W. Ed.), pp. 653-704. International edition, McGraw-Hill Publishing Company, Columbus, $\mathrm{OH}$.

Vander, A. J., Sherman, J. H. and Luciano, D. S. (1990c). The mechanisms of body function. In Human Physiology (Nunes, I., Schanck, D.T. and Bradley, J.W. Ed.), pp. 141-166. International edition, McGraw-Hill Publishing Company, Columbus, $\mathrm{OH}$. 